\title{
Diagnóstico y prevalencia de hipertensión arterial en menores de 19 años en la ciudad de Colima
}

Javier Cervantes, M.C., ${ }^{(1)}$ C uauhtémoc A coltzin, M.C., M. en C., ${ }^{(2)}$ Arnoldo Aguayo, M.C., M. en C. ${ }^{(3)}$

\section{Cervantes J, A coltzin C, A guayo A. Diagnóstico y prevalencia de hipertensión arterial en menores de 19 años en la ciudad de Colima. Salud Publica Mex 2000;42:529-532.}

\section{Resumen}

Objetivo. Estimar la prevalencia de hipertensión arterial en menores de 19 años de Colima, Colima, México. Ma terial y métodos. Estudio transversal realizado en 1992 a partir de las mediciones de la tensión arterial (TA) de 400 menores, distribuidos por sexo y edad. Se calcularon: promedio, varianza y Anova por edad; coeficientes de correlación y determinación entre edad yTA; se compararon los grupos con pruebas $t$ de Student, F y U de Mann-W hitney con Z para sexos seleccionados según la curva de distribución; asimismo, se calculó percentil 95 para definir cifras anormales. Resultados. El coeficiente de correlación es lineal, el de determinación lo confirma, se distinguen con $p<0.01$ los grupos: a) menores de dos años; b) de 2 a 13 años, y c) mayores de 13 años. Cada grupo se califica como hipertenso con cifras correspondientes de: a) $92 / 50 \mathrm{mmH}$; b) $110 / 70 \mathrm{mmH} \mathrm{g}$, y c) $133 / 84 \mathrm{mmHg}$. Conclusiones La prevalencia de hipertensión arterial en menores pudiera ser: sistólica $8 \%$ y diastólica 9\%. La hipertensión arterial resulta frecuente en los menores de edad en la ciudad de Colima y el diagnóstico es accesible.

Palabras clave: hipertensión; infante; niño; adolescencia; México
Cervantes J, A coltzin C, A guayo A.

Diagnosis and prevalence

of high blood pressure in children aged under 19

in Colima City.

Salud Publica Mex 2000;42:529-532.

\begin{abstract}
A bstract
Objective.To assess the prevalence of high blood pressure among young children. Material and methods This is a cross-sectional study, conducted in 1992 in Colima City, Mexico. Blood pressure readings were obtained from 400 children aged under 19. Statistical analysis consisted of calculation of means, variance, and Anova by group age.Association between age and blo od pressure was assessed with correlation and determination co efficients. Comparisons by sex were made using Student's t, F, Mann-W hitney's U, and $Z$ tests. Percentile $95^{\text {th }}$ was used to define normal figures. Results. A lineal correlation co efficient was found and confirmed by the determination coefficient. $G$ roups where this association was statistically significant at $p<0.01$ were: a) children aged under $2 ; b$ ) children from 2 to 13 years of age; and c) those over 13 years of age. High blood pressure figures for each group were: a) $92 / 50 \mathrm{~mm} / \mathrm{Hg} ; 110 / 70 \mathrm{~mm} /$ $\mathrm{H} \mathrm{g}$; and $133 / 84 \mathrm{~mm} / \mathrm{H} \mathrm{g}$, respectively. Conclusions The prevalence of high blood pressure in younger children may be $8 \%$ for systolic pressure and $9 \%$ for diastolic pressure.
\end{abstract}

Key words: hypertension; child, preschool; child; adolescence; Mexico

(1) Hospital G eneral de Zona c/U nidad de Medicina Familiar D octor Leonel Ramírez G arcía, Instituto Mexicano del Seguro Social (IMSS), C olima, C olima, México.

(2) Centro Universitario de Investigación Biomédica, Universidad de Colima, Colima, México.

(3) Jefatura de Enseñanza, Hospital General de Zona c/U nidad de Medicina Familiar D octor Leonel Ramírez García, IMSS, Colima, Colima, México.

Fecha de recibido: 3 de enero de 2000 - Fecha de aprobado: 22 de agosto de 2000 Solicitud de sobretiros: Cuauhtémoc A coltzin Vidal. Hospital General de Zona c/U nidad de Medicina Familiar D octor Leonel Ramírez García, Instituto Mexicano del Seguro Social. Calzada del Campesino 99, colonia El Moralete, 28060 Colima, Colima, México.

Correo electrónico: jose_rafael_c_acoltzin@yahoo.com 


\section{L} a hipertensión arterial es un padecimiento multifactorial con incidencia y prevalencia crecientes, de repercusión en órganos vitales como las arterias, corazón, riñón, entre otros, y es causa frecuente de enfermedad vascular cerebral, insuficiencia cardiaca e insuficiencia renal crónica por lo que está considerada como un problema de salud pública. ${ }^{1-3}$ Se ha informado prevalencias entre $8.3 \%$ y $22 \%$ en la población general, ${ }^{4}$ mientras que en la infantil varía entre $0.4 \%$ y $6.9 \% .^{5}$ Este estudio pretende establecer la prevalencia de hipertensión arterial en menores de 19 años de edad, en la ciudad de Colima, Colima, México.

\section{Material y métodos}

En 1992, se realizó una encuesta mediante registro de tensión arterial (TA) de 400 niños y adolescentes menores de 19 años de edad, seleccionados por cuotas, para comparar promedios, con valores alfa de 0.01 , beta de 0.95 y $d$ calculado de $1.4,{ }^{6}$ distribuidos en grupos de 10 para cada año de edad, la mitad hombres y la mitad mujeres.

Se captó a menores de cuatro años en la consulta de "control del niño sano" de la Unidad de Medicina Familiar del Hospital General de Zona Doctor Leonel Ramírez García del Instituto Mexicano del Seguro Social, de la ciudad de Colima, Colima, México, y se visitó a mayores de esa edad en escuelas públicas elegidas por azar. Se utilizó esfigmomanómetro mercurial y brazaletes de $6 \times 7 \mathrm{~cm}, 8.5 \times 20,12.5$ x 22.5, y 13.5 $x 43 \mathrm{~cm}$. Se empleó método auscultatorio en fases I y IV de Korotkoff y se siguieron las recomendaciones de la Organización Panamericana de la Salud (OPS) ${ }^{7}$ con nueve mediciones, de las que se eliminaron la 1, 4 y 7 , y se promediaron las restantes para obtener el valor medio de cada individuo.

Con valor medio por grupos se hicieron curvas, coeficientes de correlación y determinación entre edad y TA sistólica y diastólica, y se compararon con Anova. El análisis de curvas normales se hizo mediante prueba $t$ de Student; para curvas no normales, F y U de Mann-Whitney con $Z$ e intervalo de confianza del 95\% (IC 95\%) para comparar por sexo, edad y periodo de la infancia, y se graficaron. Las cifras limítrofes y anormales se definieron con curva de percentiles 90 , 95 y $99(\mathrm{P} 90=90 \mathrm{~N} / 100){ }^{8}$

\section{Resultados}

De los 400 casos, se perdieron 15 por datos incompletos, por lo que el análisis se hizo con 385 niños y adolescentes menores de 19 años. El coeficiente de correlación es lineal, es decir, muestra relación directa entre edad y cifras tensionales con valores $r=0.81$, IC $95 \% 0.67-0.90, \mathrm{~F}=72.25$ en sistólica, y $r=0.86, \mathrm{IC} 95 \%$ $0.75-0.93, \mathrm{~F}=111.01$ en diastólica.

El coeficiente de determinación lo confirma: 0.65 para sistólica, y 0.74 para diastólica. La gráfica de valores permite integrar tres grupos distintos entre sí con $p<0.001$ (figura 1): (I) menores de dos años; (II) 2 a 13 años, y (III) mayores de 13 años.

Las medias de TA, con IC 95\%, para cada grupo son: a) hombres: $70.65 \pm 7.86 \mathrm{mmHg} / 38.35 \pm 4.48$ mmHg ( $=20)$; mujeres: $78.55 \pm 2.83 \mathrm{mmHg} / 43.50 \pm$ $1.01 \mathrm{mmHg}(\mathrm{n}=18) ; \mathrm{b})$ hombres: $94.71 \pm 0.34 \mathrm{mmHg} /$ $57.77 \pm 0.96 \mathrm{mmHg}(\mathrm{n}=111)$, mujeres: $94.84 \pm 0.86$ $\mathrm{mmHg} / 56.06 \pm 0.67 \mathrm{mmHg}(\mathrm{n}=106), \mathrm{y} \mathrm{c})$ hombres: $114.59 \pm 0.82 \mathrm{mmHg} / 68.42 \pm 0.52 \mathrm{mmHg}(\mathrm{n}=60)$; $\mathrm{mu}-$ jeres: $110.71 \pm 1.27 \mathrm{mmHg} / 67.30 \pm 0.52 \mathrm{mmHg}(\mathrm{n}=70)$. No hubo diferencias entre sexos.

Las cifras limítrofe de hipertensión arterial y de gravedad, según criterio publicado a priori, $^{8}$ se muestran en el cuadro I. Las cifras anormales para cada grupo son: a) 92/50 mmHg; b) $110 / 79 \mathrm{mmHg}$, y c) $133 / 84 \mathrm{mmHg}$. La prevalencia de hipertensión arterial sistólica con cifras por arriba del percentil 95 es 8\%, de diastólica, 9\%.

\section{Discusión}

Los resultados de este estudio establecen el valor medio normal de cifras de tensión arterial de niños y adolescentes de Colima, Colima. Los coeficientes de correlación y determinación muestran que la relación

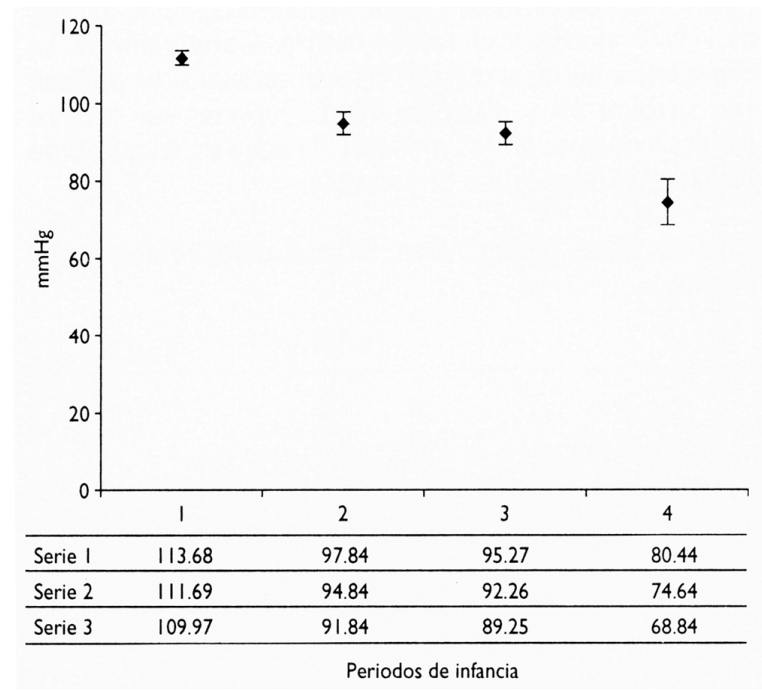

Figura 1.Tensión arterial sistólica 


\section{Cuadro I \\ DISTRIBUCIÓN DE GRUPOS LIMITROFE Y PATOLÓGICO DE CIFRAS TENSIONALES DE ACUERDO CON PERIODOS DE LA INFANCIA Y SEXO}

\begin{tabular}{lrrr}
$\begin{array}{l}\text { Clasificación } \\
\text { Percentil }\end{array}$ & $\begin{array}{c}\text { Limítrofe } \\
90 / 94\end{array}$ & $\begin{array}{c}\text { Hipertenso } \\
95 / 99\end{array}$ & $\begin{array}{c}\text { Grave } \\
>99\end{array}$ \\
$\begin{array}{l}\text { Lactante (I) } \\
\text { Femenino }\end{array}$ & $92 / 46$ & $93 / 47$ & $94 / 50$ \\
\hline Masculino & $93 / 5497 / 57$ & $100 / 56$ & \\
$\begin{array}{l}\text { Prescolar, escolar (II) } \\
\text { Femenino }\end{array}$ & $111 / 67$ & $120 / 75$ & $130 / 90$ \\
\hline $\begin{array}{l}\text { Masculino } \\
\text { Adolescente (III) } \\
\text { Femenino }\end{array}$ & $109 / 66$ & $115 / 71$ & $130 / 80$ \\
\hline Masculino & & & \\
& $126 / 79$ & $132 / 87$ & $140 / 90$ \\
\hline & $130 / 81$ & $140 / 85$ & $150 / 88$
\end{tabular}

$\mathrm{N}$ ota: las cifras tensionales se expresan en $\mathrm{mmHg}$ y se separan según propuesta de referencia9

entre edad y TA es lineal y predecible. Llama la atención que el comportamiento de la TA permite distinguir con claridad las etapas de lactante, prescolar-escolar y adolescente, pues las medias de cada grupo quedan fuera de los intervalos de confianza de los otros dos, lo que no ocurre entre prescolares y escolares, como se muestra en la figura 1.

De acuerdo con la publicación del grupo especial para el control de TA en niños ${ }^{9}$ que califica a ésta como limítrofe en percentiles 90 a 94, hipertensión arterial entre 95 y 99, y grave por arriba de 99, se han identificado las cifras que permitan el diagnóstico: 92/50 en menores de dos años; 110/70, entre 2 y 13 años, y 133/84, en mayores de 13 años.

Al contabilizar a los individuos con cifras por arriba del percentil 95 se plantea la prevalencia probable en esta muestra.

Existen otras publicaciones, como la de CobosGonzález, ${ }^{10}$ realizada en Guadalajara, que examinó 2379 niños en edad escolar y ubicó la TA en etapa IV de Korotkoff, definiendo el promedio de 105/71.95 $\mathrm{mmHg}$ para infantes de seis años y $114.88 / 74.70 \mathrm{mmHg}$ para los 12 años, y la de Moreno-Altamirano, ${ }^{11}$ que examinó niños semejantes de la ciudad de México y definió el valor medio de $91 / 61 \mathrm{mmHg}$ para niños de seis años y 102/68 mmHg para los de 12 años. Ningún autor encontró diferencia según sexo. Este trabajo aportó cifras tensionales esperadas en menores de dos años de edad y en adolescentes.
Se partió del registro ocasional de TA, sin investigar daño en órganos blanco ni sancionar el pronóstico a largo plazo, lo que impide afirmar cronicidad de la elevación tensional, pero se informan cifras promedio de seis mediciones, conforme la recomendación de la OPS, distinto de las obtenidas en una sola, pues se elimina la variación. Además, se acepta que el registro de TA diastólica en lactantes resulta poco confiable ya que la identificación de la etapa IV es difícil.

Este hallazgo difiere del de recién nacidos de otros países, ${ }^{9}$ quienes han sido clasificados por meses de edad y comparados por percentiles obtenidos mediante curva de regresión. Sin embargo, es nuestra apreciación que la prevalencia de hipertensión arterial sistólica de $8 \%$ y diastólica de $9 \%$ resulta probable.

Se establecen los límites de normalidad y del estado supuestamente patológico en tres etapas de la infancia, pero el diagnóstico de hipertensión arterial sistémica y la decisión de tratamiento se deberán apoyar en criterios de daño de órganos blanco y cronicidad, entendida como el resultado de cifras anormales en múltiples mediciones realizadas en diferentes fechas. ${ }^{12, *}$

La información obtenida mejorará la atención médica de los menores, ayudará en el diagnóstico y al manejo de la hipertensión arterial y facilitará la detección de padecimientos como lupus eritomatoso sistémico, enfermedad de Takayasu, hipoplasia renal y uropatía obstructiva.

\section{Referencias}

1. Stokes J, Kannel W B,W olf PA, C upples LA, D 'A gostino RB. Importancia relativa de los factores de riesgo que se relacionaron para manifestaciones diversas de la actividad cardiovascular entre hombres y mujeres de 35 a 64 años de edad: seguimiento de 30 años en el estudio Framingham. Hypertension 1992;3:27-37.

2. Frolich ED. Hipertensión. En: Lewis RP, Crawford MH, Gunnar RM, ed. Programa de autoformación clínica en adultos. México, D.F.: A merican College of $\mathrm{C}$ ardiology, American Heart A sociation, American College of Physicians, Consejo Mexicano de Cardiología 1994;6:6.3.

3.C hávez-D omínguez R,A coltzin-Vidal C,C arboney-C astellanosA, LópezFranchini J, Moragrega-A dame JL, Rendón-Muñiz J. D efinición, causas, cla-

* Subsecretaría de Prevención y Control de Enfermedades. Coordinación de Vigilancia Epidemiológica. Proyecto de Norma Oficial Mexicana para la Prevención, Tratamiento y Control de la Hipertensión Arterial. México, D.F.: SSA, abril de 2000. Documento no publicado. 
sificación, epidemiología, tasas de mortalidad. I Consenso nacional de hipertensión arterial. Rev Mex Cardiol 1995;6:S10-S14.

4. Hernández LM, A coltzin $C$, Enríquez RD. Factores de riesgo coronario en varones sujetos a stress. Rev Med Inst Mex Seguro Soc 1992; 30:351-359.

5. Hernández-Martínez MA, Torres-Pastrana J, Manrique-N ájera AM, Pezzotti-y Rentería MA. Hipertensión arterial en pediatría. Bol Med Hosp Infant Mex 1989;46:60-64.

6. C añedo-D orantes L. Investigación clínica. México, D.F.: N ueva Editorial Interamericana, 1987:178-183.

7.Voors AW, Foster T, Frerichs R, W ebbwe L, Berenson G. Studies of blood presure in children, ages 5-14 years, in a total biracial community. The Bogalusa Heart Study. Circulation 1976,54:319-327.

8. C astilla-Serna L, C ravioto J. Estadística simplificada para la investigación en ciencias de la salud. México, D.F:: Editorial Trillas, 1991:26-58.
9. Task force on blood pressure control in children. Report of the second task force on blood pressure control in children-1987. Pediatrics 1987; 79:1-25.

10. C obos 0 , Rubio R, García-de Alba JE, Parra JZ. La presión arterial en escolares de Guadalajara. Salud Publica Mex 1983;25:177-183.

11. Moreno-Altamirano L, Kuri-Morales P, Guémez-Sandoval JC,VillazónSalem S.Tensión arterial en escolares de la ciudad de México. Importancia de las tablas de valores normales. Bol Med Hosp Infant Mex 1987;44: 389-395.

12. O rganización Mundial de la Salud. Sociedad Internacional de Hipertensión. Lineamientos para el tratamiento de la hipertensión. Aterosclerosis 1999;2(2):34-52 